\title{
Title:
}

\section{The diagnostic value of capsule endoscopy in children with intestinal lymphangiectasia}

\section{Authors:}

Jie Wu, Zhiheng Huang, Min Ji, Zhinong Jiang, Yuhuan Wang, Zifei Tang, Ying Huang

DOI: $10.17235 /$ reed.2021.7682/2020

Link: PubMed (Epub ahead of print)

Please cite this article as:

Wu Jie, Huang Zhiheng, Ji Min, Jiang Zhinong, Wang Yuhuan, Tang Zifei, Huang Ying. The diagnostic value of capsule endoscopy in children with intestinal lymphangiectasia. Rev Esp Enferm Dig 2021. doi:

$10.17235 /$ reed.2021.7682/2020.

This is a PDF file of an unedited manuscript that has been accepted for publication. As a service to our customers we are providing this early version of the manuscript. The manuscript will undergo copyediting, typesetting, and review of the resulting proof before it is published in its final form. Please note that during the production process errors may be discovered which could affect the content, and all legal disclaimers that apply to the journal pertain. 
The diagnostic value of capsule endoscopy in children with intestinal lymphangiectasia

Jie $\mathrm{Wu}^{1}$, Zhiheng Huang ${ }^{1}$, Min $\mathrm{Ji}^{2}$, Zhinong Jiang ${ }^{3}$, Yuhuan Wang ${ }^{1}$, Zifei Tang ${ }^{1}$, Ying Huang ${ }^{1}$

1. Gastroenterology Department/Endoscopy Centre, Children's Hospital of Fudan University, Shanghai, China 201102

2. Department of Radiology, Children's Hospital of Fudan University, Shanghai, China 201102

3. Department of Pathology, Sir Run Run Shaw Hospital, Zhejiang University School of Medicine, Hangzhou, Zhejiang, China

Correspondence to: Ying Huang, MD, PhD

Department of Gastroenterology, Children's Hospital of Fudan University, Shanghai, China

E-mail: yhuang815@163.com

OR to Zifei Tang, MD

Department of Gastroenterology, Children's Hospital of Fudan University, Shanghai, China

E-mail: 82401328@qq.com

Conflicts of Interest: None

Declaration of Funding Source: None

List of abbreviations: CT: computed tomography, IL: intestinal lymphangiectasia, MRI: magnetic resonance imaging, VCE: video capsule endoscopy,

Keywords: video capsule endoscopy, children, intestinal lymphangiectasia, hypoalbuminemia

\footnotetext{
Abstract

Background: Intestinal lymphangiectasia is an unusual cause of protein-losing enteropathy due to either congenital malformation or the obstruction of the
} 
intestinal lymphatics. However, few reports have investigated the use of video capsule endoscopy in children with intestinal lymphangiectasia. This study was performed to evaluate the diagnostic value of video capsule endoscopy for paediatric intestinal lymphangiectasia.

Methods: In this retrospective study, we included all patients who underwent video capsule endoscopy between January 2014 and July 2020. Clinical information and video capsule endoscopy data were analysed.

Results: Twelve children, 7 males and 5 females, with an age of disease onset of 4.5 (range: 3.2-9.3) years, and a disease duration of 12.0 (range: 1.3-30.0) months were enrolled. The most common symptoms were hypoproteinaemia (10, 83.3\%), diarrhoea (7, 58.3\%), oedema $(6,50.0 \%)$, and abdominal pain $(3,25.0 \%)$. Eight patients had low lymphocyte counts, whereas 10 had reduced serum albumin levels $(23.2 \pm 5.8 \mathrm{~g} / \mathrm{L})$. Video capsule endoscopy revealed an overall white snowy appearance due to the presence of whitish, swollen villi in all patients. Regarding the macroscopic lesions of lymphangiectasia, 7 cases involved the entire small bowel from the duodenum to the ileocecal valve, while 5 cases involved part of the small bowel. All patients were treated with medium-chain triglyceride diets, and albumin infusions were administered for 10 patients; sirolimus treatment was administered to 3 patients. At the last follow-up, 5 patients still had hypoalbuminemia, and one patient had died of intestinal lymphoma.

Conclusion: Video capsule endoscopy is useful for the diagnosis of intestinal lymphangiectasia and should be applied as a valuable and less invasive examination to confirm or establish a diagnosis. 


\section{Introduction}

Intestinal lymphangiectasia (IL) is a rare disease characterized by dilatation and rupture of intestinal lymphatic channels, leading to protein-losing enteropathy[1]. IL is defined as the dilation of existing mucosal, submucosal, or subserosal lymphatics within the gastrointestinal tract. Furthermore, IL causes the loss of lymph fluid into the gastrointestinal tract, resulting in the development of hypoproteinaemia, oedema, lymphocytopaenia, hypogammaglobinaemia, and immunologic anomalies[2]. IL is easily diagnosed by upper gastrointestinal endoscopy and confirmed by pathology if the lesions involve the duodenum. However, the presence of patchy areas of lymphangiectasia in the jejunum and ileum cannot be detected because of the length limitation on upper gastrointestinal endoscopy. Thus, it is necessary to explore the entire small bowel to determine the extent of lymphangiectasia.

The development of video capsule endoscopy (VCE) was a milestone in gastroenterological endoscopy. The United States Food and Drug Administration approved the use of CE for the evaluation of small bowel diseases in adults in 2001[3]. In 2004, VCE was approved for children 10 years or older. Overall, VCE is an important diagnostic tool for the signs of small bowel diseases, such as patchy areas of lymphangiectasia in the jejunum and ileum[4, 5].

The diagnosis of IL via VCE in paediatric patients has been documented in only a few case series[6]; in general, data are lacking, especially for Chinese children. This study was performed to analyse the clinical features and describe the feasibility and value of the use of VCE for the diagnosis of IL, shedding new light on the clinical application of VCE.

\section{Materials and methods}

This retrospective study was approved by the Ethics Committee of the Children's Hospital of Fudan University. Informed consent for participation was obtained from the parents or legal guardians of the patients. In this study, we included all patients who were diagnosed with IL who underwent VCE between January 2014 and July 2020. 
The OMOM capsule endoscopy system, with a diameter of $11.0 \mathrm{~mm}$ and a length of $25.4 \mathrm{~mm}$, used in this study was purchased from Jinshan Science \& Technology (Chongqing, China). The intestinal preparation procedure was described previously[7]. The diagnosis of IL was made through upper gastrointestinal endoscopy, VCE and histological pathology. Clinical information, VCE data and follow-up data were analysed.

\section{Results}

\section{Patient characteristics}

From January 2014 to July 2020, 12 children were diagnosed with IL by VCE at the Children's Hospital of Fudan University; 7 were male, and 5 were female. Among these patients, the age of disease onset was 4.5 (range: 3.2-9.3) years, and the disease duration was 12.0 (range: $1.3-30.0$ ) months. No patients had a family history of IL.

\section{Clinical and laboratory data}

The clinical features of the patients varied. The most common symptoms were hypoproteinaemia $(10,83.3 \%)$, diarrhoea $(7,58.3 \%)$, oedema $(6,50.0 \%)$, and abdominal pain $(3,25.0 \%)$ (Table 1). Ascites and pleural effusion were detected by ultrasound in 4 patients.

According to laboratory tests, 8 patients had low lymphocyte levels, and 10 had reduced levels of serum albumin $(23.2 \pm 5.8 \mathrm{~g} / \mathrm{L})$ (Table 2). Furthermore, 5 patients had anaemia, with haemoglobin levels ranging from $98 \mathrm{~g} / \mathrm{L}$ to $110 \mathrm{~g} / \mathrm{L}$.

To detect a possible infectious aetiology of $\mathrm{IL}$, additional investigations were performed. However, tests for human immunodeficiency virus (HIV), hepatitis C virus (HCV), hepatitis B virus (HBV), herpes simplex, rubella virus and parasites were negative, while case 3 was positive for Epstein-Barr virus (EBV) DNA.

\section{VCE and imaging data}

Gastroscopic assistance for capsule introduction was needed in 9 out of the 12 children. In all children, the capsule was expelled in the stool, and no adverse effects occurred. The average small-bowel transit time was $331.6 \pm 184.8 \mathrm{~min}$. 
VCE showed an overall white, snowy appearance due to the presence of whitish, swollen villi in all cases. Seven children had macroscopic lesions of lymphangiectasia involving the entire small bowel from the duodenum to the ileocecal valve (Figure $1 A-C)$; in five patients, only part of the small bowel was affected (Figure D-F). All patients underwent upper gastrointestinal endoscopy before VCE, and 7 were suspected of having IL based on the presence of macroscopic lesions of lymphangiectasia (Figure H). Seven patients underwent enteroscopy, and 3 were suspected of having IL based on the presence of macroscopic lesions of lymphangiectasia (Figure I). IL was confirmed by the histologic analysis of duodenal and jejunal biopsy specimens that showed dilated intestinal lymphatics (Figure 2). Six patients were diagnosed with IL by duodenal biopsy after gastrointestinal endoscopy. Five patients with a normal duodenum underwent enteroscopy to obtain jejunal biopsies. Moreover, magnetic resonance imaging (MRI) and computed tomography (CT) showed thickening of the walls of the jejunum and ileum (Figure 3 ).

\section{Treatment and follow up}

All patients were treated with a medium-chain triglyceride (MCT) diet; albumin infusions were administered to 10 patients because of severe hypoproteinaemia. Ten patients underwent follow-up, and 2 were lost to follow-up. Case 3 died of intestinal T-cell lymphoma. Case 12 was diagnosed with mesenteric lymphangioma. Cases 9, 10 and 12 were administered sirolimus treatment. At the last follow-up, 5 patients still had hypoproteinaemia and needed hospital follow-up and albumin infusions regularly.

\section{Discussion}

This study reports the characteristics of 12 children with IL who underwent small bowel examination by VCE. IL may be characterized as a disorder involving dilated intestinal lacteals causing the loss of lymph into the lumen of the small intestine and resulting in hypoproteinaemia, hypogammaglobulinaemia, hypoalbuminaemia and reduced numbers of circulating lymphocytes or lymphopaenia[8].

Mucosal lesions can be detected in the duodenum of children during gastroscopic examination if IL is suspected. However, for lesions below the horizontal part of the 
duodenum, jejunum and ileum, enteroscopy and VCE are needed[9]. Overall, VCE can aid in achieving a diagnosis when patients have distal disease of the small bowel. We performed a literature review of IL patients diagnosed by VCE. In 2006, P Chamouard diagnosed two authentic adult cases of protein-losing enteropathy using VCE, showing that the technique is useful in cases of protein-losing enteropathy to enable the identification of the presence of intestinal lymphangiectasia and to specify the localization after ruling out other disorders liable to induce protein-losing gastrointestinal syndromes[4]. We have found several articles regarding the usefulness of VCE in children with IL $[6][10,11]$. The first series was published by Christine et al and included 4 children aged 6, 10, 15 and 17 years old. Our study involved 12 children diagnosed with IL by VCE, including 5 with partial small bowel involvement and 7 with complete small bowel involvement.

The diagnosis of IL is confirmed if diffuse snow-white dots, villous prominence and chyle are observed in the small bowel mucosa[12]. A high-fat meal challenge before endoscopy can improve the diagnostic yield. Pathology shows the dilation of existing mucosal, submucosal, or subserosal lymphatics within the gastrointestinal tract[1, 13]. For the diagnosis of primary IL, secondary factors, such as autoimmune disease, tumour and infectious diseases (tuberculosis, parasites), should be excluded[14-16]. Endoscopic examination and duodenal biopsy are standard criteria for the diagnosis of intestinal lymphangiectasia.

For patients with hypoproteinaemia, it is reasonable to begin the evaluation with upper endoscopy or colonoscopy. Furthermore, VCE should be performed if the underlying aetiology remains uncertain or a small bowel aetiology is suspected. VCE offers a relatively easy way to view the mucosa of the entire small bowel, to make an educated guess based on the gross appearance and to exclude other disorders possibly responsible for protein loss[13].

IL is often associated with diarrhoea and abnormal laboratory findings. The main laboratory findings in IL are reduced lymphocyte counts and calcium ion, serum albumin, and gamma globulin (IgA, IgG, IgM) levels. History, physical examination, and laboratory data are important clues to the diagnosis of IL[17]. Moreover, nutritional treatment is very important, and a low-fat diet with supplementary MCT 
is the cornerstone of the medical management of primary IL [18].

Our study had limitations. VCE could not be used in more IL patients due to difficulty swallowing the device. Lymphoscintigraphy is another efficient way to detect intestinal lymphangiectasia. Unfortunately, there are no lymphoscintigraphy instruments in our hospital because of the limited resources. Therefore, lymphoscintigraphy could not be performed.

Conclusions: Both primary and secondary causes of IL should be evaluated to determine the appropriate management of affected patients. VCE may be useful for diagnosing IL and determining the extent of the lesions and the appropriate followup.

\section{References}

1. Vignes, S. and J. Bellanger, Primary intestinal lymphangiectasia (Waldmann's disease). Orphanet J Rare Dis, 2008. 3: p. 5.

2. Oh, T.G., et al., Primary intestinal lymphangiectasia diagnosed by capsule endoscopy and double balloon enteroscopy. World J Gastrointest Endosc, 2011. 3(11): p. 235-40.

3. Glukhovsky, A. and H. Jacob, The development and application of wireless capsule endoscopy. Int J Med Robot, 2004. 1(1): p. 114-23.

4. Chamouard, P., et al., Videocapsule endoscopy is useful for the diagnosis of intestinal lymphangiectasia. Dig Liver Dis, 2006. 38(9): p. 699-703.

5. Wu, J., et al., Clinical features of capsule endoscopy in 825 children: A singlecenter, retrospective cohort study. Medicine (Baltimore), 2020. 99(43): p. e22864.

6. Rivet, C., et al., Use of capsule endoscopy in children with primary intestinal lymphangiectasia. Gastrointest Endosc, 2006. 64(4): p. 649-50.

7. $\mathrm{Wu}, \mathrm{J}$, , et al., The diagnostic value of video capsule endoscopy for Meckel's diverticulum in children. Rev Esp Enferm Dig, 2020. 112.

8. Prasad, D., et al., Clinical Profile, Response to Therapy, and Outcome of Children with Primary Intestinal Lymphangiectasia. Dig Dis, 2019. 37(6): p. 458-466. 
9. Arguelles-Arias, F., et al., Guideline for wireless capsule endoscopy in children and adolescents: A consensus document by the SEGHNP (Spanish Society for Pediatric Gastroenterology, Hepatology, and Nutrition) and the SEPD (Spanish Society for Digestive Diseases). Rev Esp Enferm Dig, 2015. 107(12): p. 714-31.

10. Antao, B., et al., Clinical application and diagnostic yield of wireless capsule endoscopy in children. J Laparoendosc Adv Surg Tech A, 2007. 17(3): p. 364-70.

11. van der Reijden, S.M., et al., Video Capsule Endoscopy to Diagnose Primary Intestinal Lymphangiectasia in a 14-Month-Old Child. J Pediatr Gastroenterol Nutr, 2017. 64(6): p. e161.

12. Ohmiya, N., et al., Classification of intestinal lymphangiectasia with proteinlosing enteropathy: white villi type and non-white villi type. Digestion, 2014. 90(3): p. 155-66.

13. Ersoy, O., et al., Evaluation of primary intestinal lymphangiectasia by capsule endoscopy. Endoscopy, 2013. 45 Suppl 2 UCTN: p. E61-2.

14. Patel, K.V., R.M. Goel, and T. Wong, Diffuse large B-cell lymphoma recurrence complicating primary intestinal lymphangiectasia. Clin Gastroenterol Hepatol, 2013. 11(12): p. e86-7.

15. Ozen, A., et al., CD55 Deficiency, Early-Onset Protein-Losing Enteropathy, and Thrombosis. N Engl J Med, 2017. 377(1): p. 52-61.

16. Hoshina, T., et al., Cytomegalovirus-associated protein-losing enteropathy resulting from lymphangiectasia in an immunocompetent child. Jpn J Infect Dis, 2009. 62(3): p. 236-8.

17. Troskot, R., et al., How to treat an extensive form of primary intestinal lymphangiectasia? World J Gastroenterol, 2015. 21(23): p. 7320-5.

18. Li, S., et al., Nutritional therapy and effect assessment of infants with primary intestinal lymphangiectasia: Case reports. Medicine (Baltimore), 2017. 96(51): p. e9240. 
Table 1 : Clinical features of IL patients.

\begin{tabular}{ll}
\hline Patients & $\mathrm{N}=12$ \\
\hline Sex (male: female) & $7: 5$ \\
Age of admission (years) & $7.1 \pm 3.8$ \\
Age of disease onset (years) & $4.5[3.2,9.3]$ \\
Duration time (months) & $12.0[1.3,30.0]$ \\
Symptoms & \\
Diarrhoea & 8 \\
Oedema & 8 \\
Abdominal distension & 6 \\
Abdominal pain & 5 \\
Ascites and pleural effusion & 4 \\
Anaemia & 5 \\
Vomiting & 2 \\
\hline
\end{tabular}

Table 2: Laboratory findings in IL patients. 


\begin{tabular}{|c|c|}
\hline Laboratory test & Value \\
\hline Lymphocyte $\left(\times 10^{\wedge} 9 / L\right)$ & $1.77 \pm 0.97 \quad(1.2-4)$ \\
\hline Albumin (g/L) & $23.50 \pm 5.66 \quad(40-55)$ \\
\hline Prealbumin (mg/L) & $216.58 \pm 63.30(200-400)$ \\
\hline $\mathrm{Ca} 2+(\mathrm{mmol} / \mathrm{L})$ & $2.01 \pm 0.23(2.2-2.6)$ \\
\hline $\lg G(g / L)$ & $4.41 \pm 2.28(6.98-14.26)$ \\
\hline $\lg A(g / L)$ & $0.59 \pm 0.38(0.92-2.5)$ \\
\hline $\lg M(g / L)$ & $0.72 \pm 0.40(0.56-2.16)$ \\
\hline Triglycerides(mmol/L) & $1.07 \pm 0.49(0-1.7)$ \\
\hline Cholesterol(mmol/L) & $3.54 \pm 1.10(0-5.18)$ \\
\hline
\end{tabular}



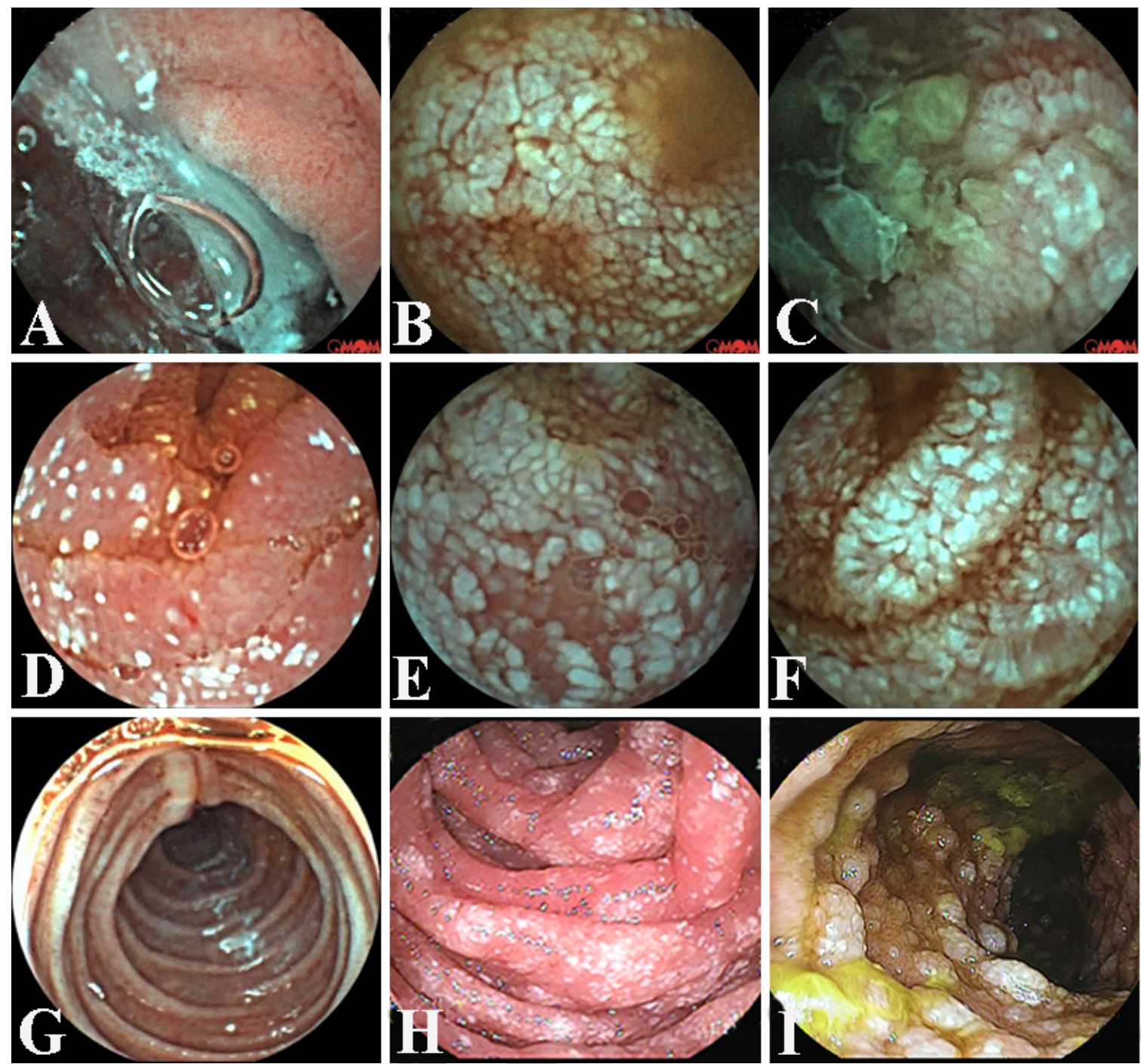

Figure 1. VCE image. Whitish, swollen villi were seen in the duodenum (A), jejunum (B) and ileal mucosa (C) of case 11. Images of mild (D), and moderate-severe $(E, F)$ lymphangiectasis in cases 2, 9 and 12, respectively. No whitish patches in the duodenum of case $4(\mathrm{G})$. The image of duodenum $(\mathrm{H})$ and terminal ileum (I) after gastrointestinal endoscopy and enteroscopy examinations in case 11. 


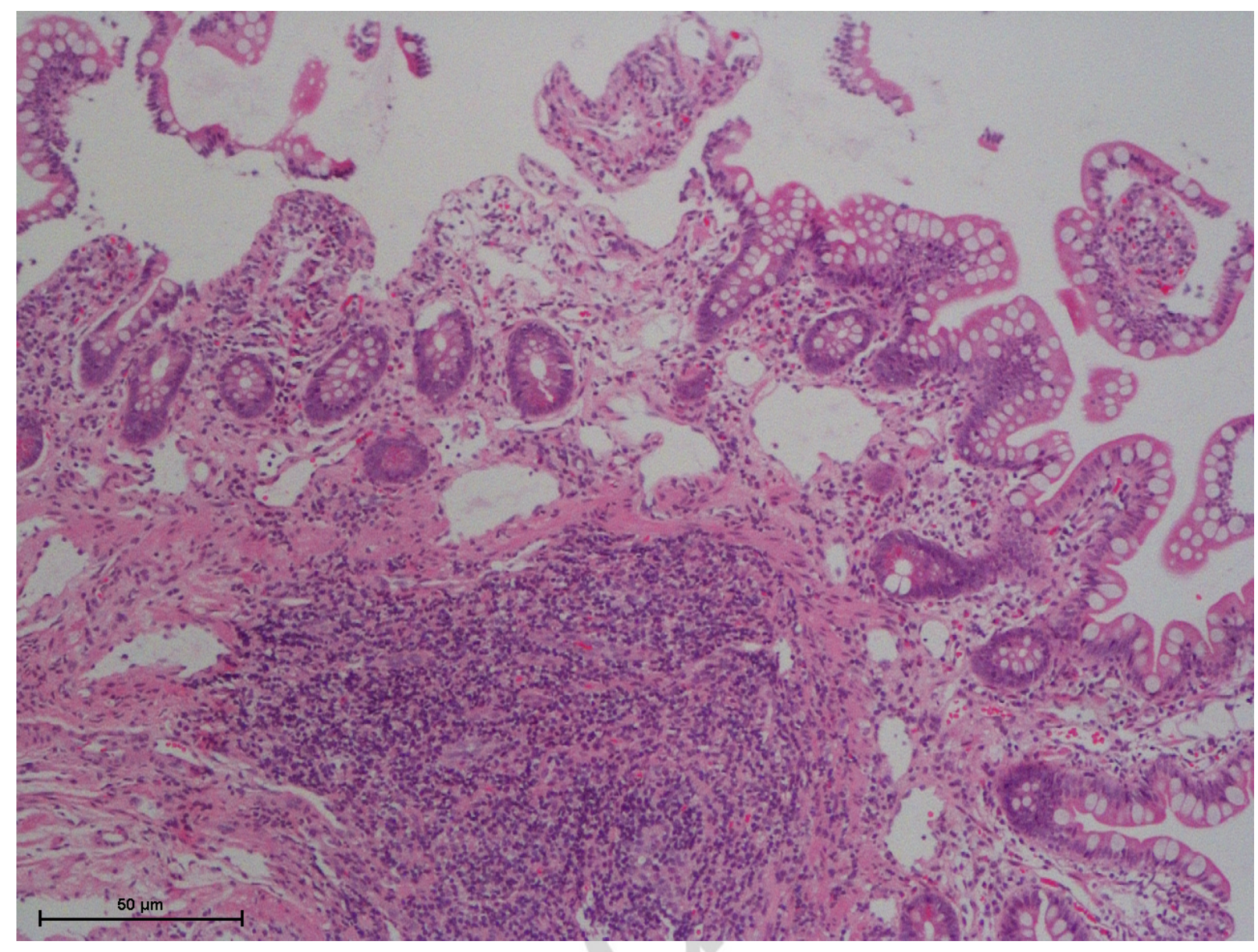

Figure 2. Duodenal biopsy showing a markedly dilated lymphatic duct in case 12. 

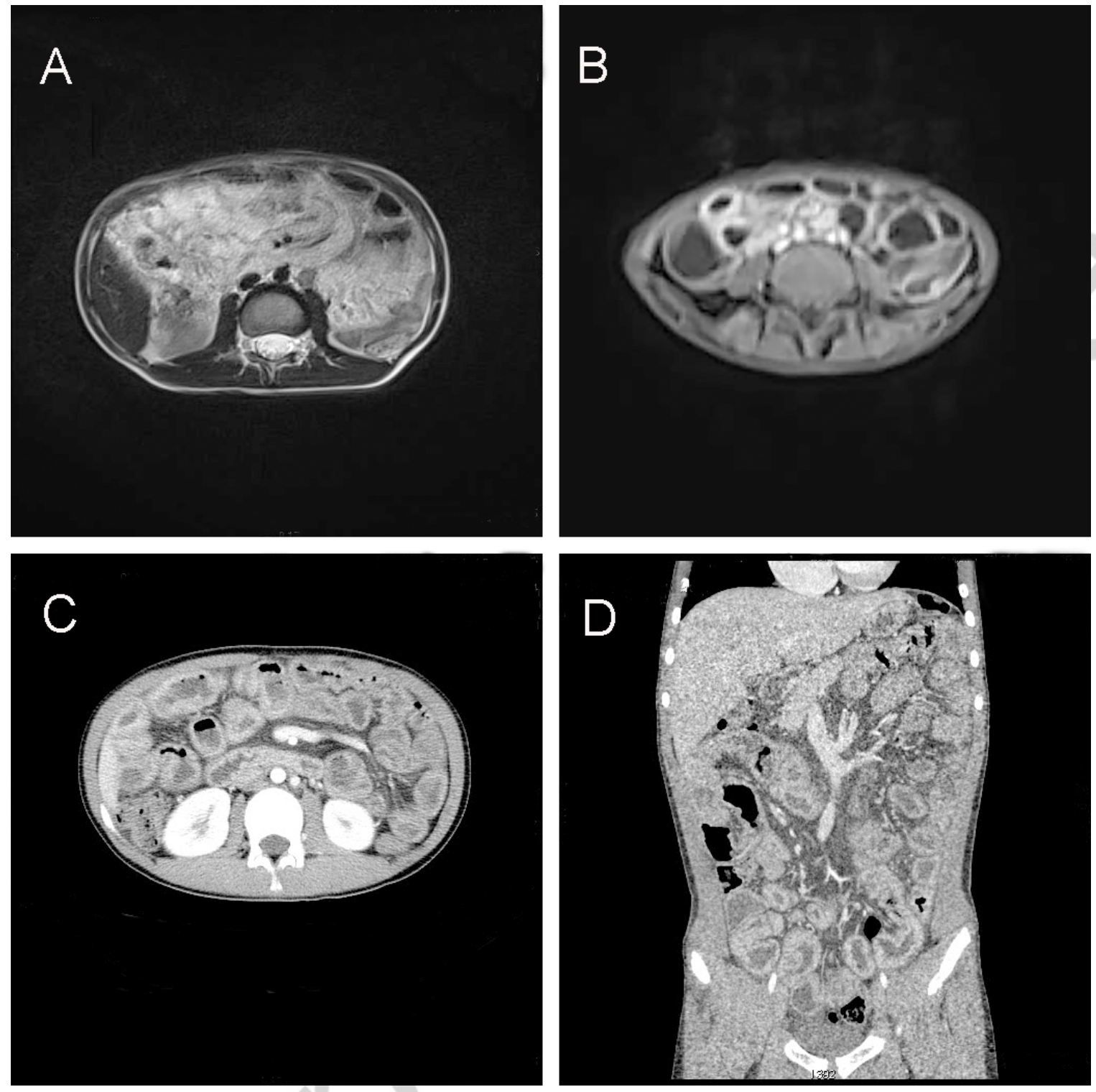

Figure 3. MRI and CT images showing thickening of the walls of the jejunum and ileum in cases 12 (A, B) and 10 (C, D), respectively. 